\title{
Performance Research on Iterative Methods for Image Deblurring
}

\author{
Mahendra B M, Savita Sonoli
}

\begin{abstract}
This paper introduces the iterative image restoration algorithms for the elimination of linearly varying blurs from the images degraded by motion blur and additive noise. Iterative algorithms are very operative for this applications since they include different types of prior knowledge about the class of reasonable solutions. These algorithms are robust in nature to the errors in estimating the blurring operators and can be used to remove the non-stationary blurs. Performance analysis and limitations of traditional approaches such as Inverse, Wiener and Constrained Least Square filters (CLS) are discussed with respect to the iterations. Role and choice of imposing a constraint on the solutions of the algorithms which gives better restoration results are discussed. Regularization methods are debated to Minimis extreme noise amplifications due to ill-posed conditions in the inverse deblurring problems and it is shown that the reduction of noise effects can be achieved by terminating the algorithm after finite number of iterations. It is shown that restoration algorithms with constraints and spatially adaptability reduces the effects of ringing artifacts significantly. The rate of convergence of the algorithms based on the variations in the number of iterations are discussed and performance analysis, limitations and Comparison with the experimental results are presented.
\end{abstract}

Index Terms - Algorithm, Adaptable, Blur, Constrained, Comparison, Convergence, Deblurring, Restoration, Iterative, Ill posed, Image, Least Squares, Performance, Regularization, Ringing, Spatially.

\section{INTRODUCTION}

The modern imaging sciences getting more attention towards the motion deblurring, owing to be very common in the areas of remote sensing, medical imaging, inspection, surveillance, astronomy, autonomous vehicle guidance, and more [1-5]. A quick with long, decisive steps are made in developing efficient algorithms in this specific field. It is well known that the main cause of image blurring is the alteration in grey values of image pixels or the change in positions of pixels from its original points due to the relative motion between the object and the camera during image capturing.

This paper discusses the restoration of blurred images using iterative approaches. In recent history iterative restoration problems are very active in the research field of image restoration [6-18]. It has been continuously proved that these iterative approaches can be exclusively powerful due to the following reasons 1 . Inverse of an operator is not can be used in restoration process 3 . Solution of the process is controlled and monitored 4. Effect of the noise can be controlled with the help of constraints. In this papers many

Revised Manuscript Received on July 10, 2019.

Mahendra B M, RV College of Engineering, Bengaluru, Karnataka India. (Email: mahen.8787@gmail.com)

Dr. Savita Sonoli, Rao Bahadhur Y Mahabaleshwarappa Engineering College, Bellary, Karnataka India. (Email: savitachitriki@gmail.com) necessary to implement 2. Prior knowledge of the solution

of such modern improvements and advantages of iterative algorithms in restoring images are explained.

This paper is organized into several divisions. The solutions to an ill conditioned problem [20], [22] is highly sensitive to the noise in an image restoration. Numerous of the early solutions were concerned with this problem and these classical approaches such as inverse, wiener or least squares (LS) filters [20], [22] and constrained least squares (CLS) [6], [13], [20], [25] solutions are discussed in section I. The potential advantage of iterative image restoration which can be used in straight forward way of image restoration is discussed in section II. The major interest of using iterating approaches is the fact that the restoration process is monitored as it progresses, therefore it is possible to terminate the process at any point where the results are satisfactory. In continuation with the iterative approaches the concept of regularization which resolves ill-conditioned problems with insensitive to measurement noise and the rate of convergence of the algorithms based on the variations in the number of iterations are discussed. The effect of noise can be controlled in each iteration. Both iterative and noniterative regularized restoration algorithms are explained along with numerous illustrations are given which clearly prove the power of the method. In section III the spatial [19], [28] adaptive restoration filter is discussed with experimental results, where the restoration filter takes the local content of the image into account which increases smoothness and reduces the effects of ringing artifacts [15] comparatively.

\section{TRADITIONAL APPROACHES}

\section{A. Inverse Filter}

The continuous domain representation of the degraded image $g(\alpha, \beta)$ in terms of input image $f(x, y)$ assuming the blur is linear and time invariant is written as a convolution of the input image and Point Spread Function[1-5] i.e.

$$
g(\alpha, \beta)=\int_{-\infty}^{+\infty} \int_{-\infty}^{+\infty} f(x, y) h(x, \alpha, y, \beta) d x d y
$$

where $h(x, \alpha, y, \beta)$ is the PSF (Point Spread Function) that expresses the degradation effect.

Ideally the blur model is [1] represented as

$$
g=f \otimes h
$$

The Fourier Transform (FT) of the above equation is represented as

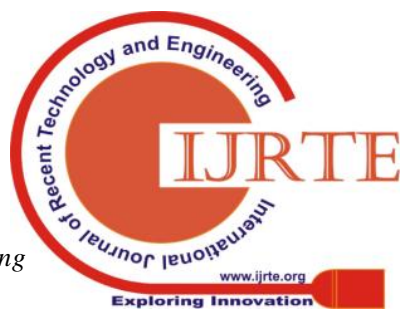




$$
\hat{G}(u, v)=\hat{F}(u, v) \cdot \hat{H}(u, v)
$$

If we have a prior knowledge of PSF or its FT of the blurring process the image deblurring [1] problem may be solved. The information of the PSF can be obtained from the knowledge of the physical process like diffraction, atmospheric turbulence, or from the image data itself that caused the blur.

If $H(u, v)$ is very small value or does not contain 0 and the blurred image is noise-free, the latent image $F(u, v)$ can be found by inverting the convolution process using inverse filtering, the easiest method that solves for $F(u, v)$. i.e.

$$
\hat{F}(u, v)=\frac{\hat{G}(u, v)}{\hat{H}(u, v)}
$$

Ideally the frequency contents of $\hat{H}(u, v)$ and $\hat{G}(u, v)$ becomes zero at some point in the ${ }^{(u, v)}$ plane, then the ratio $\hat{G}(u, v)$

$\overline{\hat{H}(u, v)}$ will be undetermined i.e. for the specific frequencies $(u, v)$ the frequency contents of original image cannot be restored. This problem can be overcome by neglecting corresponding points in the frequency plane.

During image capturing process, the Gaussian, multiplicative, Poisson and impulse noise effects the image along with the blur and leads to loss of information. The image with the blur along with the noise is modelled as

$$
\begin{aligned}
& g(\alpha, \beta)=\int_{-\infty}^{+\infty} \int_{-\infty}^{+\infty} f(x, y) h(x, \alpha, y, \beta) d x d y+n(x, y) \\
& g=f \otimes h+n
\end{aligned}
$$

The Fourier Transform of the above equation is represented as

$$
\begin{aligned}
& \hat{G}(u, v)=\hat{F}(u, v) \hat{H}(u, v)+N(u, v) \\
& \hat{F}(u, v)=\frac{\hat{G}(u, v)}{\hat{H}(u, v)}-\frac{N(u, v)}{\hat{H}(u, v)}
\end{aligned}
$$

If the blur model is having the least amount of noise, even if the numerator is tremendously small and when the denominator turns out to be 0 , the result is tremendously large, i.e. frequencies destroyed by the imaging process will actually be extremely amplified hence slight amount of noise saturates the inverse filter.

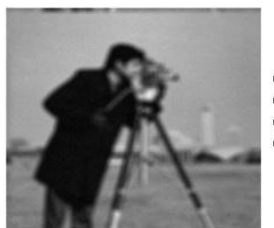

(a)

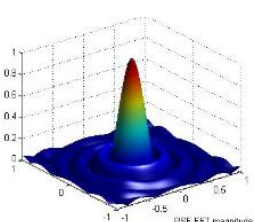

(b)

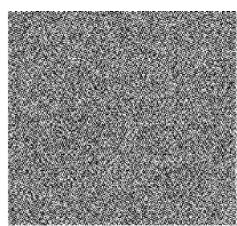

(c)
Fig.1. (a) Synthetic blurred image with circular averaging filter with radius 4 with PSNR 40dB. (b)Frequency response of PSF.(c)Restored image.

\section{B. Wiener Filter or Least Square Filter}

To minimise the sensitivity of noise in the inverse filter shown in Fig.1. (a), a number of restoration filters have been introduced [23] which will be collectively called as leastsquares (LS) filters. The linear space-invariant Wiener filter makes use of the power spectrum of both the image and the noise to avoid amplification of noise. The frequency response of this filter $H(u, v)$, is chosen to lessen the mean squared restoration error (MSE) $\varepsilon$, given by

$$
\begin{gathered}
\varepsilon=\mathrm{E}\left(|F(u, v)-\hat{F}(u, v)|^{2}\right) \\
\varepsilon=\mathrm{E}\left(|F(u, v)-\hat{G}(u, v) \hat{M}(u, v)|^{2}\right)
\end{gathered}
$$

where $E(\mathrm{~g})$ represents the expectation over an ensemble of images. The response to this minimization problem is given by

$$
\hat{M}(u, v)=\frac{H^{*}(u, v)}{|H(u, v)|^{2}+\frac{S_{n n}(u, v)}{S_{\pi}(u, v)}}
$$

Where $S_{f f}(u, v)$ is the power spectrum of the original image, $\operatorname{Snn}(u, v)$ is the power spectrum of the noise, and $H^{*}(u, v)$ denotes the complex conjugate of $H(u, v)$ At frequencies $(u, v)$ where the signal is much stronger than the noise, the ISNR is 0 , and the Wiener filter reduces to inverse filter. At frequencies $(u, v)$ where the signal is much weaker, the ISNR will be large and the corresponding component $G(u, v)$, will be attenuated.

The Wiener filter in the noiseless case approximates the pseudo-inverse filter defined by

$$
\hat{M}_{P S}(u, v)= \begin{cases}\frac{1}{\hat{H}(u, v)} & \text { for } H(u, v) \neq 0 \\ 1 & \text { for } H(u, v)=0\end{cases}
$$

A Wiener filter restoration is shown in Fig.2.

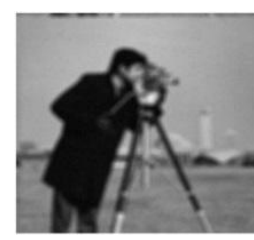

(a)

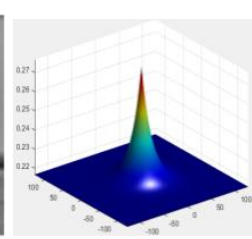

(b)

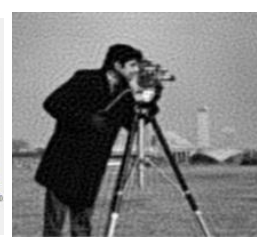

(c)
Fig.2. (a) Synthetic blurred image with circular averaging filter with radius 4 with PSNR 40dB (b) Frequency response of PSF (c) Restored image with improvement in the SNR 5.90 dB

The extreme noise amplification of the previous example is exists no longer because of masking of spectral zeros, but the image is still somewhat blurred. It has been a regular criticism of Wiener filters is that they mainly act to suppress measurement noise, while carrying out only slight deblurring.

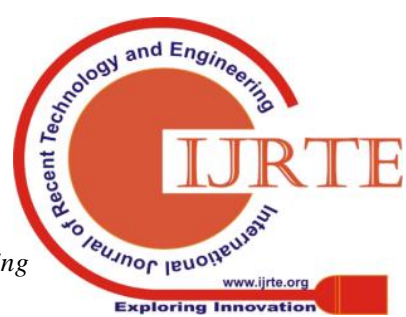


If the statistical properties of the image are unknown then restoration can be done by replace the term $\operatorname{Svv}(\mathrm{u}, \mathrm{v}) / \mathrm{Sff}$ $(\mathrm{u}, \mathrm{v})$ in equation (13) with a constant $\tau$ and experiment with various values of the constant

$$
\hat{M}(u, v)=\frac{H^{*}(u, v)}{|H(u, v)|^{2}+\tau}
$$

\section{Constrained Least Square(CLS) Filters}

CLS filtering is an alternative method for avoiding difficulties related with the inverse filter, by holding the simplicity of using a single Linear Space Invariant (LSI) filter to restore the image [6], [13], [24], [25].

The wiener filter requires the knowledge of power spectral properties of noise and the undegraded image. It is shown that wiener filter is possible to get the satisfactory results but finding powers spectral ratio is not a feasible solution. CLS Solution requires only the knowledge of mean and variance of the noise and these two parameters can be calculated from the given degraded image.

It is most commonly used image restoration technique. It leads to unconstrained to constrained least square problems. Finding prior knowledge to find the best solution is not difficult. Prior knowledge is Lagrange multiplier or regularisation parameter. This method is nothing but impose a constraint on the solution

$$
\begin{aligned}
& \text { Minimize } J(f)=\|y-H f\|^{2} \\
& \text { Subjected to }\|C f\|^{2}<\varepsilon
\end{aligned}
$$

Where $\mathrm{C}$ is the operator that needs to be chosen i.e. High Pass Filter. The constraint $\|C f\|^{2}<\varepsilon$ gives the energy imposed by the norm of the restored image at high frequencies is below the threshold $(\varepsilon)$, which means it doesn't allow the arbitrary high frequency fluctuations in the solution or differently it imposes a smoothness requirement on the solution.

The standard way to solve the constraint problem is to relax it and turn it unconstrained problem by introducing constrained parameter $\operatorname{alpha}(\alpha)$ which is regularisation parameter or Lagrange multiplier. The regularisation is the process of not allowing the noise amplification i.e.

$$
\underset{\mathrm{f}}{\operatorname{Min}}\left(\|y-H f\|^{2}+\alpha\|C f\|^{2}\right)
$$

Now it is necessary to minimize the function inside the parenthesis by taking the gradient of it and equate it to zero i.e. unconstrained gradient operator. By solving equation (18), the solution gives the expression

$$
f=\left(H^{T} H+\alpha C^{T} C\right)^{-1} H^{T} y
$$

if $\mathrm{H}^{\mathrm{T}} \mathrm{H}$ is not invertible then the term $\alpha \mathrm{C}^{\mathrm{T}} \mathrm{C}$ makes $\mathrm{H}^{\mathrm{T}} \mathrm{H}$ invertible or if $\mathrm{H}^{\mathrm{T}} \mathrm{H}$ is large then it is called ill-posed then the term $\alpha \mathrm{C}^{\mathrm{T}} \mathrm{C}$ makes the term $\mathrm{H}^{\mathrm{T}} \mathrm{H}$ smaller and hence the problem behaves better.

The common choice of operator $\mathrm{C}$ is a high pass filter, such as 2D Laplacian i.e. impulse response of the 2D Laplacian is

$$
\left[\begin{array}{ccc}
0 & 1 & 0 \\
\hline 1 & -4 & 1 \\
0 & 1 & 0
\end{array}\right]
$$

If $\mathrm{H}$ and $\mathrm{C}$ are block circulant matrix, then the expression of constrained least square filter is given by

$$
\hat{M}(u, v)=\frac{H^{*}(u, v)}{|H(u, v)|^{2}+\alpha|C(u, v)|^{2}}
$$

Clearly if $\alpha=0$, then the constrained least square becomes lease square inverse filter. If the solution of CLS filter is a function of $\alpha$, then $\alpha$ is chosen in such a way that

$$
\|C f\|^{2}<\varepsilon
$$

Assuming that the value of $\mathcal{E}$ is known, then by adjusting $\alpha$ the constraint can be satisfied. The effect of $\alpha$ with PSNR $=40 \mathrm{~dB}$ and noise power is zero on the performance of CLS Filter is shown in Fig.3.

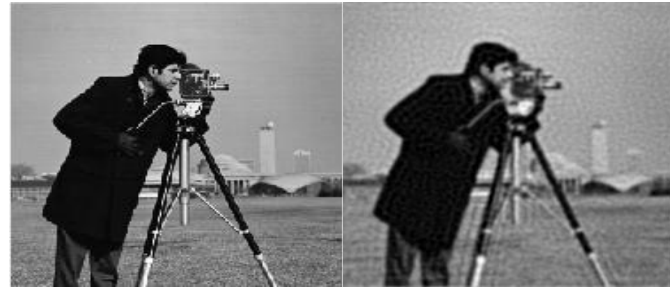

(a)

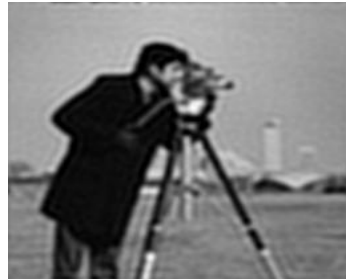

(c) (b)

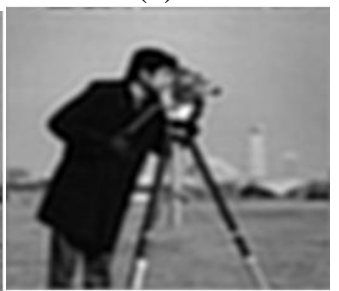

(d)
Fig.3. (a) Original image (b) $\alpha=0.01$ (c) $\alpha=0.1$ (d) $\alpha=1.0$

It is evident from the Fig.3. is that a smaller $\alpha$ results in a noisier but sharper image while larger alpha results in smoother but blurrier image. In this case the improvement in SNR is $6.2 \mathrm{~dB}$.

The choice of $\alpha$ is very important, since it gives the tradeoff between the noise amplification and smoothness of the filter. The best way of choosing $\alpha$ is based on the two parameters i.e. variance and bias from the original and restored image.

\section{ITERATIVE APPROACHES}

Iterative algorithms provide solutions to nonlinear but very often to linear optimisation problems as well. In this session we discuss the potential advantage of iterative image restoration which can be used in straight forward way of image restoration [6-18]. The formulation also allows constraints during the iteration process which incorporates prior information of the original image to the solution

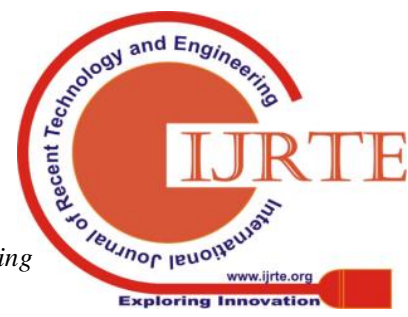


process. Using a straight forward approach analysis, it is possible to derive the sufficient condition for the convergence of the algorithm [9]. Some of the advantages of iterative algorithms are, inverse filters are implemented iteratively instead of implementing the filter directly. The restoration process is monitored as it progress, therefore it is possible to terminate the process at any point where the results are satisfactory. The effect of noise can be controlled in each iteration, hence more the iterations higher the noise amplification. These methods can be applied to spatially varying or nonlinear degradations, for the type of degradation is unknown.

\section{A. Van Cittert's approach}

Let the degradation equation

$$
g=f \otimes h+n
$$

the simplest possible way of finding $f$ is of the form

the successive approximation iterations form shown here

$$
f_{k+1}=f_{k}+\beta\left(y-H f_{k}\right)=\beta y+(I-\beta H) f_{k}
$$

It is a rather straight forward iteration to implement [18-]. Where $\beta \mathrm{H}$ is independent of iterations and $\mathrm{I}-\beta \mathrm{H}$ is a matrix and $\mathrm{fk}$ is the previously restored image [32]. Finding all above and simplifying gives the next better restored image. If the system is LSI, $\mathrm{H}$ is block circulant matrix, then iterations (25) can be taken in DFT domain. i.e.

$$
F_{k+1}(u, v)=\beta Y(u, v)+(1-\beta H(u, v)) F_{k}(u, v)
$$

Now it is necessary to find the sufficient condition for the convergence of the algorithm. Let

$$
F_{k}(u, v)=R_{k}(u, v) Y(u, v)
$$

Where $R_{k}(u, v)$ is Iteration Filter, $Y(u, v)$ is DFT of the degraded image, $F_{k}(u, v)$ is $\mathrm{K}^{\text {th }}$ restored image. The iteration process will start by considering

$$
\begin{gathered}
F_{0}(u, v)=0 \\
F_{1}(u, v)=\beta Y(u, v) \\
F_{2}(u, v)=\beta Y(u, v)+(1-\beta H(u, v)) F_{1}(u, v) \beta Y(u, v) \\
+(1-\beta Y(u, v)) \beta Y(u, v) \\
F_{3}(u, v)=\beta Y(u, v)+(1-\beta H(u, v)) \beta Y(u, v) \\
+(1-\beta H(u, v)))^{2} \beta Y(u, v)
\end{gathered}
$$

Here $F_{3}(u, v)$ is a $1^{\text {st }}$ order differential equation with respect to the index ' $\mathrm{k}$ '. It is clearly observed that the restoration filter is the $\mathrm{K}^{\text {th }}$ iteration step i.e.

$$
R_{k}(u, v)=\beta \sum_{i=0}^{k-1}(1-\beta H(u, v))^{l}
$$

If $k \rightarrow \infty$ the magnitude of the term $1-\beta Y(u, v)$ strictly less than 1 . Hence this indeed sufficient condition for the convergence i.e. if

$$
|1-\beta H(u, v)|<1
$$

If $\mathrm{H}(u, v)$ is complex then the Region Of Convergence (ROC) is shown in Fig.4.

$$
\phi(f)=y-H f
$$

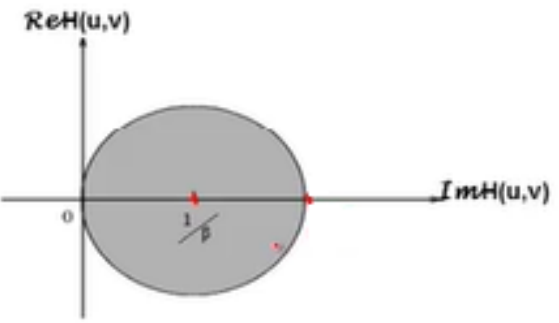

Fig.4. The region of convergence

If $\mathrm{H}$ is real and non-negative, then

$$
0<\beta<\frac{2}{H_{\max }(u, v)}
$$

Where $H_{\max }(u, v)=1_{\text {hence the degradation is }}$ normalized.

If $\mathrm{H}(u, v)$ is negative, then equation (33) doesn't satisfies. By considering the limits for the filter

$$
\begin{aligned}
& \lim _{k \rightarrow \infty} R_{k}(u, v)=\lim _{k \rightarrow \infty} \beta \frac{1-(1-\beta H(u, v))^{k}}{1-(1-\beta H(u, v))}
\end{aligned}
$$

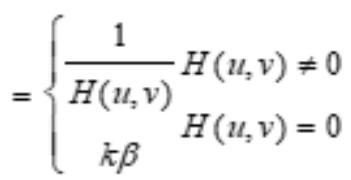

$$
\begin{aligned}
& \text { If } \quad k \rightarrow \infty \text {,the term } 1-\beta H(u, v) \rightarrow 0
\end{aligned}
$$

since $|1-\beta H(u, v)|<1$. For $\mathrm{k} \rightarrow \infty$ or those frequencies $\mathrm{H}(u, v) \neq 0$ and the filter acts as inverse filter and for the frequencies $\mathrm{H}(\mathrm{u}, \mathrm{v})=0$, the filter acts as $\mathrm{k} \beta$. This will be the major difference between generalised inverse filter and iterative inverse filter. It is observed that, this restoration takes place per frequency component independently that means, for the frequencies where $\mathrm{H}$ is small, the converges will be slow and at frequencies where $\mathrm{H}$ is large, then the converges will be faster i.e. the lower frequencies could be restored faster than the high frequencies.

\section{B. Iterative Least Square Method or Gradient Decent Method:}

In this segment we utilize the successive approximations or iterative approach towards the solutions of least squares filters. It is a gradient decent method in a sense since we are utilising gradient of the objective function although we are utilising fixed step size. We show sufficient condition for convergence of the iterations. Demonstrated experimentally an important benefit of the iterative restoration algorithms over a direct implementation of the restoration filters i.e. the number of iterations can be used as a means of regularisation. That is to control the trade of between sharpness of the solution and noise amplifications. Due to this, the choice of regularisation parameter also becomes secondary, i.e. the value of regularisation parameters can be chosen based on some general guidelines and then the most desirable solution can be obtained with the appropriate number of iterations. 
Consider the least square iteration function and the successive approximation iterations

$$
\begin{gathered}
\phi(f)=\frac{1}{2} \nabla_{f}\|y-H f\|^{2} \\
f_{k+1}=f_{k}+\beta H^{T}\left(y-H f_{k}\right) \\
f_{k+1}=\beta H^{T} y+\left(I-\beta H^{T} H\right) f_{k}
\end{gathered}
$$$$
\text { Where } H^{T}(y-H f) \text { is gradient of the norm. If } \mathrm{H} \text { is }
$$
block circulant matrix, then equation (37) in DFT domain is $F_{k+1}(u, v)=\beta H^{*}(u, v) Y(u, v)+\left(1-\beta|H(u, v)|^{2}\right) F_{k}(u, v)$

The convergence properties are obtained by expressing the filter in the form

$$
\begin{gathered}
R_{k}(u, v)=\beta \sum_{i=0}^{k-1}\left(1-\beta|H(u, v)|^{2}\right)^{l} H^{*}(u, v) \\
R_{k}(u, v)=\beta \frac{1-\left(1-\beta|H(u, v)|^{2}\right)^{k}}{1-\left(1-\beta|H(u, v)|^{2}\right)} H^{*}(u, v)
\end{gathered}
$$

The convergence of this series when $k \rightarrow \infty$ i.e. sufficient condition for convergence is:

$$
0<\beta<\frac{\left.|1-\beta| H(u, v)\right|^{2} \mid<1}{\max |H(u, v)|^{2}}
$$

$|\mathrm{H}(u, v)|$ is always real and non-negative and limiting condition for restoration filter is given by

$$
\begin{aligned}
\lim _{k \rightarrow \infty} R_{k}(u, v) & =\lim _{k \rightarrow \infty} \beta \frac{1-\left(1-\beta|H(u, v)|^{2}\right)^{k}}{1-\left(1-\beta|H(u, v)|^{2}\right)} H^{*}(u, v) \\
& =\left\{\begin{array}{c}
\frac{1}{H(u, v)} H(u, v) \neq 0 \\
0(u, v)=0
\end{array}\right.
\end{aligned}
$$

It is observed that, it exactly works like a general inverse filter but, the process is depending on the number of iterations. Standard convergence criteria called residual

$$
\text { error is given by } \frac{\left\|f_{k+1}-f_{k}\right\|^{2}}{\left\|f_{k}\right\|^{2}}
$$

The residual error versus number of iterations for the iterative least square algorithm with $1 \mathrm{D}$ motion blur over 8 pixels with no noise is given as

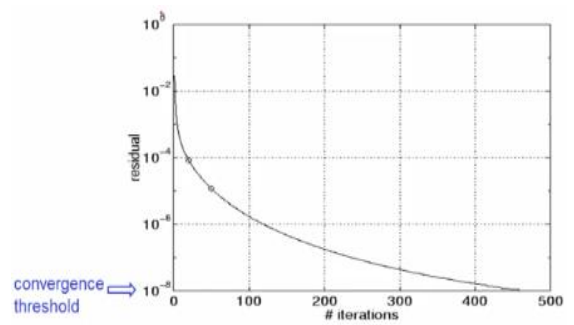

Fig.5. Residual error v/s Number of iteration.

The system is said to be convergence when the residual error becomes smaller than the threshold. Convergence threshold is set at $10^{-8}$ and the system converges at $465^{\text {th }}$ iterations. The experimental results with respect to different iterations until the system converges is shown in Fig.6.

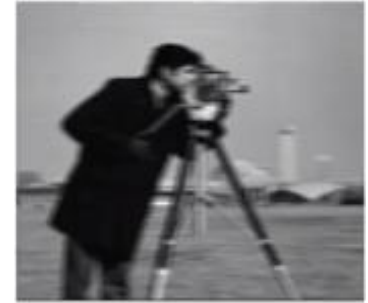

(a)

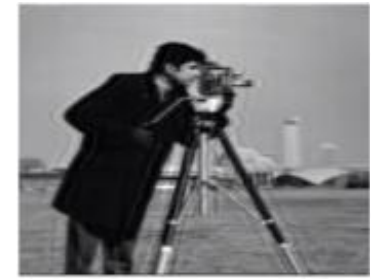

(c)

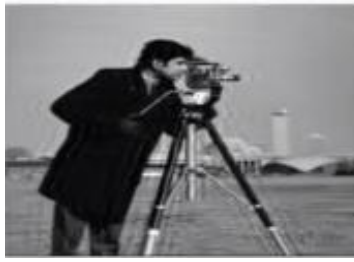

(e)

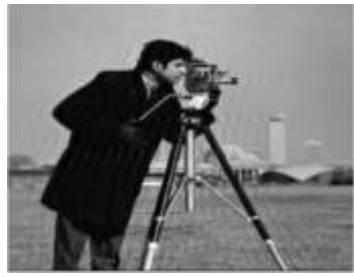

(g)

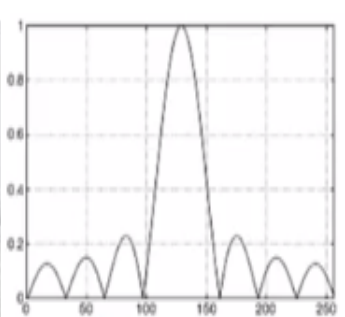

(b)

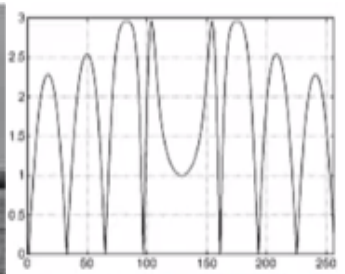

(d)

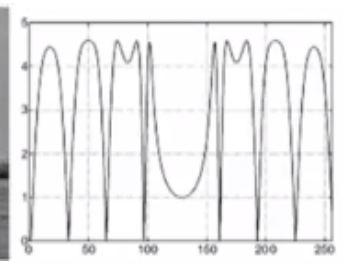

(f)

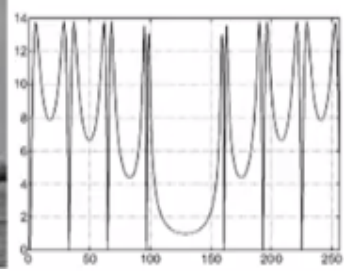

(h)

Fig.6. (a) 1D motion blur over 8 pixels with no noise.

(c)K=20,ISNR=4.03dB. $(\mathrm{e}) \mathrm{K}=50, \mathrm{ISNR}=6.22 \mathrm{~dB}$ (g)K=465,ISNR=11.58 dB. (b),(d),(f) and (h) Magnitude of frequency response of degradation system.

Consider an image degraded with 1 dimensional motion blur over 8 pixels with no noise and slice of magnitude frequency response of a degradation system shown in Fig.6. (a) and (b). (c) is the restored image from iterative LS filter $\mathrm{K}=20$ iterations with $\mathrm{ISNR}=4.03 \mathrm{~dB}$, (d) is the restored image with $\mathrm{K}=50, \mathrm{ISNR}=6.22 \mathrm{~dB}$. (e) is restored image at $\mathrm{K}=465$, ISNR $=11.58 \mathrm{~dB}$. From the observation the general statement by ISNR values from the visual quality, as more the iteration, sharper the restored image and this agrees that low frequencies are restored faster than the high frequencies so as the iterations progresses, sharper and sharper edges in the restored image.

Comparing the two images restored from the iterative LS filter at number of iterations equal to 465 and with direct inverse filter, the ISNR $=11.58 \mathrm{~dB}$ in the latter case and

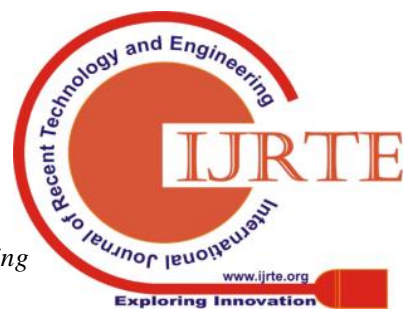


ISNR $=15.50 \mathrm{~dB}$ in the present case. Based on the metrics, the direct inverse filtered image is closed to the original image, but by visual quality it is arguable that the restored image by direct inverse filter shows more pronounced ringing artifacts than the image restored from iterative LS Filter which is shown in Fig.7.

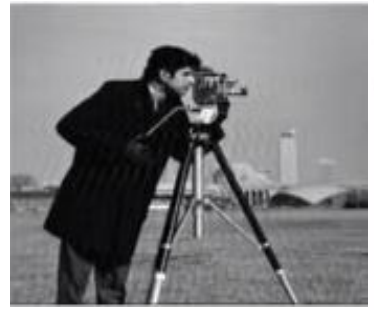

(a)

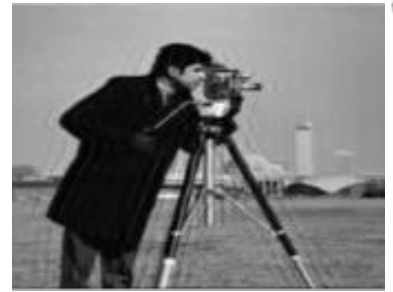

(c)

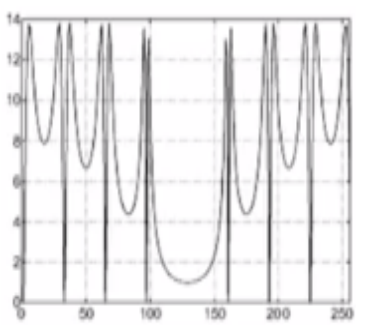

(b)

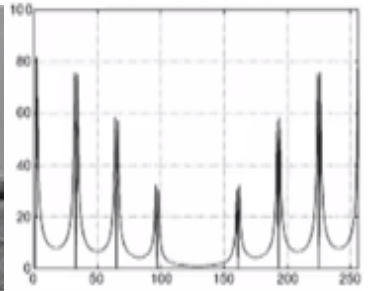

(d)
Fig.7. (a)Iterative LS filter restored image with $K=465$, ISNR=11.58dB. (c)K=465, ISNR=15.50dB. (b), (d)Magnitude of frequency response of degradation system.

The point to be noted is, the fact that error based objective metrics of quality do not really always represent the visual quality of the images. By observing the frequency response of the restoration filter from Fig.7. (b) (d), the iterative LS Filters tapering off at high frequencies results in good visual quality of output image with the presence of noise. Actually, this is the property that really provides advantages to the iterative restoration filters when the noise is present. This method gradually restores high frequencies of the image iteration by iteration. The behaviour of iterative method in presence of both blur and noise is shown in Fig.8. with 1D motion blur over 8 pixels with BSNR=20dB.

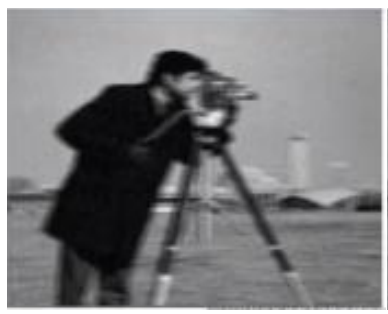

(a)

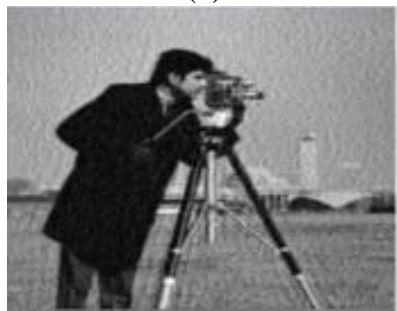

(c)

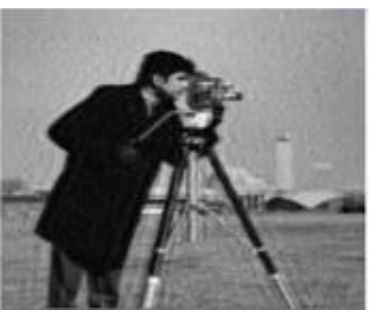

(b)

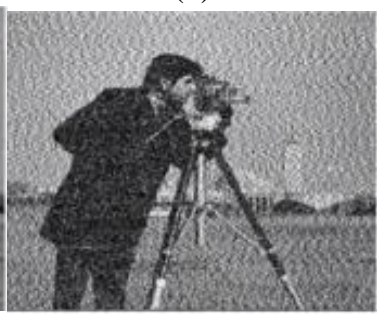

(d) (e)

Fig.8. (a) 1Dimentional motion blur over 8 pixels with BSNR=20dB (b) $K=20, I S N R=1.83 \mathrm{~dB}$ (c) $K=50$, ISNR = -0.30dB (d) K=1376, ISNR=-9.06 dB(e) Direct inverse filter with $\mathrm{ISNR}=\mathbf{- 1 2 . 0 9} \mathrm{dB}$.

It is concluding from the observation of restoration results shown in Fig.8. the iterative implementation of filters performing better in restoration to the noise amplification. however, the real advantage come from the fact that in getting to the restoration at $\mathrm{K}=1376$ iterations of convergence, any of the better restoration results between $\mathrm{K}=20$ and 50 can be picked. At $\mathrm{K}=50$ and above, acceptable decent restoration results can be expected. This method restores high frequencies but at the same time it amplifies the noise and it takes place gradually iteration by iteration, therefore the number of iteration provides a means of regularisation. the noise amplification can be controlled by choosing any intermediate iteration results before the convergence as they restore the images. By visual quality inspection the restored image with $\mathrm{ISNR}=-0.3 \mathrm{~dB}$ is better than ISNR $=-12.09 \mathrm{~dB}$.

\section{Iterative Constrained Least Square Filter:}

In addition to the fidelity term, a smoothness constraint $\|\mathrm{Cf}\|^{2}$ and $\alpha$ i.e. regularisation parameter is included to the function $\phi(f)$. then

$$
\phi(f)=\frac{1}{2} \nabla_{f}\left(\|y-H f\|^{2}+\alpha\|C f\|^{2}\right)
$$

The resulting iteration using successive approximation

$$
F_{k+1}=\beta H^{T} y+\left(I-\beta\left(H^{T} H+\alpha C^{T} C\right)\right) f_{k}
$$

When $\mathrm{C}$ and $\mathrm{H}$ are block circulant then in DFT domain the above equation is written as

$$
\begin{aligned}
& F_{k+1}(u, v)=\beta H^{*}(u, v) Y(u, v)+ \\
& \left(1-\beta\left(|H(u, v)|^{2}+\alpha|C(u, v)|^{2}\right)\right) F_{k}(u, v)
\end{aligned}
$$

The resulting convergence analysis in frequency domain is

$$
R_{k}(u, v)=\beta \sum_{i=0}^{k-1}\left(1-\beta\left(|H(u, v)|^{2}+\alpha\|C f\|^{2}\right)\right)^{l} H^{*}(u, v)
$$

and the sufficient condition for the convergence is

$$
\left|1-\beta\left(|H(u, v)|^{2}+\alpha|C(u, v)|^{2}\right)\right|<1
$$

If $\mathrm{H}(\mathrm{u}, \mathrm{v})=0$ at some points then $\mathrm{C}(\mathrm{u}, \mathrm{v}) \neq 0$ and satisfies the conditions of convergence. 
As $\mathrm{K} \rightarrow \infty$, The limiting condition for restoration filter is given by

$$
\lim _{k \rightarrow \infty} R_{k}(u, v)=\left\{\begin{array}{cc}
\frac{H^{*}(u, v)}{H(u, v)^{2}+\alpha|C(u, v)|^{2}} & |H(u, v)|^{2}+\alpha|C(u, v)|^{2} \neq 0 \\
0 & |H(u, v)|^{2}+\alpha|C(u, v)|^{2}=0
\end{array}\right.
$$

Experimental results of iterative CLS filter considering the image degraded due to motion blur with 8 pixels and $\mathrm{BSNR}=20 \mathrm{~dB}$ with $2 \mathrm{D}$ Laplacian and $\alpha=0.01$ is used and the restored image $\mathrm{ISNR}=2.12 \mathrm{~dB}$ at $20^{\text {th }}$ iterations and ISNR= 0.98 at $50^{\text {th }}$ Iteration is achieved and shown in Fig.9. (a) and (b) respectively. It is observed that ISNR is decreased with increasing number of iterations i.e. the number of iterations increases, the edges become sharper and noise multiplication takes place. But still noise amplification is acceptable, compared to the latter approaches.

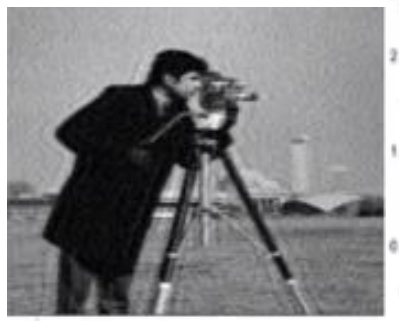

(a)

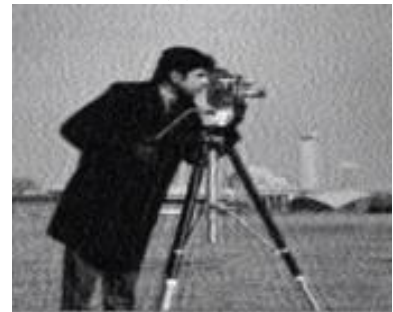

(c)

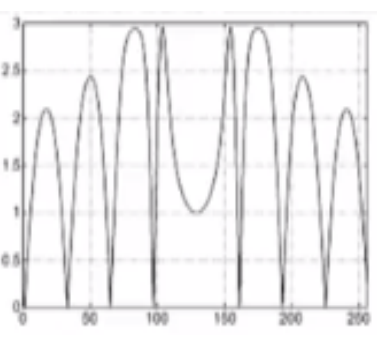

(b)

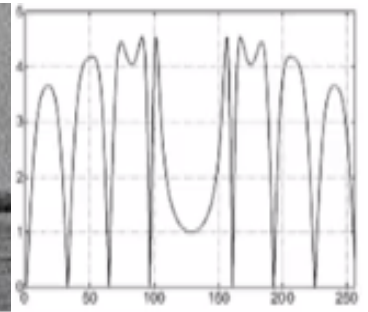

(d)
Fig.9.(a) Restored image, $K=20$, ISNR=2.12dB (c) Restored image, $\mathrm{K}=50$, ISNR=0.98dB (b), (d) Magnitude of frequency response of degradation system.

Comparison between the direct CLS filter and iterative CLS filter by again considering an image degraded by horizontal motion with 8 pixels and $B S N R=20 \mathrm{~dB}$ is shown in Fig.10. Iterative CLS filter is having better ISNR shown in (a) with $\mathrm{K}=330, \mathrm{ISNR}=-1.01 \mathrm{~dB}$ and the restored image by directs CLS filter shows poor performance with ISNR= $1.64 \mathrm{~dB}$ shown in (b)

In magnitude plot of direct CLS filter it is observed that at some frequencies, the high frequency values converge slowly but at the same points with iterative CLS filter the values will be smaller and converges faster.

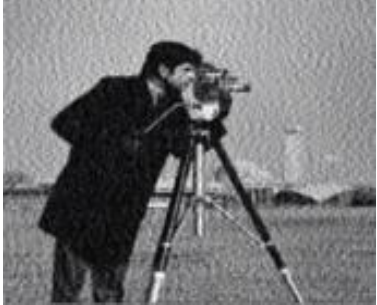

(a)

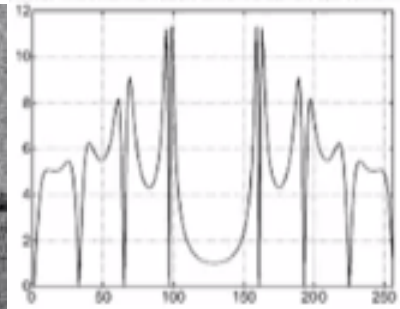

(b)

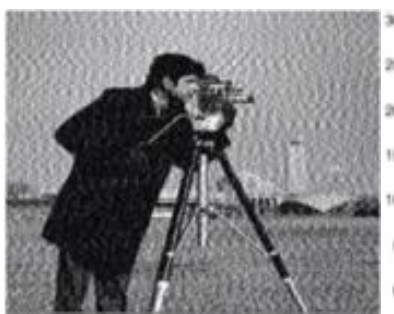

(c)

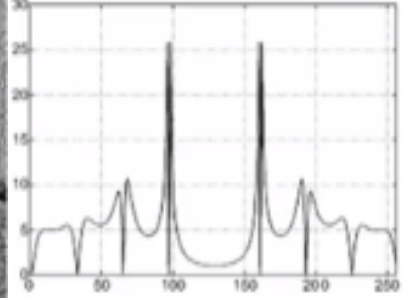

(d)
Fig.10.(a) Iterative CLS filtered restored image with K=330, ISNR=-1.01dB. (c) Directs CLS filtered restored image with $K=330$, ISNR=-1.64dB. (b)(d) Magnitude of frequency response of degradation system.

\section{SPATIALLY ADAPTIVE ALGORITHMS:}

Improved restorations can be obtained, when the restoration filter takes the local content of the image into account. In this method a different restoration filter is applied to each and every pixel [19]. In this section we explain the spatial adaptive restoration filter with the use of weighted norms and a specific way to adjust these weights based on human visual system.

One way to introduce special adaptivity for the constrained least square filters is to consider the function $\phi(f)$, which we want to find its roots is given by

$$
\phi(f)=\frac{1}{2} \nabla_{f}\left(\|y-H f\|_{w 2}^{2}+\alpha\|C f\|_{w 2}^{2}\right)
$$

Where $\|y-H f\|_{w 2}^{2}$ is the norm of fidelity to the data term with weight $\mathrm{W} 1$ and $\|C f\|_{w 2}^{2}$ is the norm of smoothness constraint with weight $\mathrm{W} 2$. The weights $\mathrm{W} 1$ and $\mathrm{W} 2$ are interpreted as it is know that the weighted norm of $\mathrm{X}$ is given by

$$
\begin{gathered}
\|X\|_{w}^{2}=X^{T} W^{T} W_{X} \\
\|X\|_{W}^{2}=\sum_{i=1}^{N} W_{i}^{2} X_{i}^{2}
\end{gathered}
$$

Where $\mathrm{W}$ is the Diagonal matrix given by

$$
W=\left[\begin{array}{lll}
\pi_{1} & & \\
& w_{2} & \\
& & \pi_{N}
\end{array}\right]
$$

It is observed that the weights $\mathrm{W}$ gives different importance to elements $\mathrm{X}$ and $\mathrm{Wi}>0$ which is non negative. The successive approximation based iteration is given by $F_{k+1}(u, v)=\beta H^{T} W_{1}^{T} W_{1} y+\left(I-\beta\left(H^{T} W_{1}^{T} W H+\alpha C^{T} W_{2}^{T} W_{2} C\right)\right) f_{k}$

If $\mathrm{W} 1=\mathrm{W} 2=\mathrm{I}$ then the above filter is exactly equal to iterative CLS filter.

The above term cannot be represented as DFT domain even though $\mathrm{H}$ and $\mathrm{C}$ are block circulant since W1 and W2 are not block circulant and hence these filters can be implemented in spatial domain. Convolutions are performed in discrete frequency domain and then we can take the

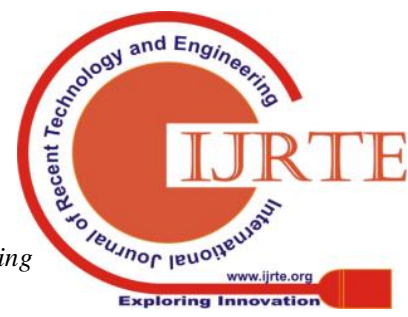


results back to the spatial domain to implement their weights and go back and forth. Some choices of choosing the weights are as follows

$$
\text { Let } W_{2}=V
$$

Where $\mathrm{V}=$ visibility matrix and is given by

$$
V \approx \frac{1}{\sigma_{f}^{2}}
$$

Where $\sigma_{f}^{2}$ gives the local variance of the image.

According to the masking properties of the human visual system, high frequency information in the image masks high frequency noise i.e. the noise is not visible at the edges as in the flat regions in an image. Therefore, one of the forms of the restoration, we can allow noise go through at the edges and this can be accomplishing by setting the entries of the matrix $\mathrm{W}$ are very small close to zero at the edge locations, which disables the smoothness constraint and allow them to be large at flat regions which enables the smoothness constraint. Hence visibility matrix can be chosen as shown in the equation (57). At the edges, local variance is large and leads to $\mathrm{W} 2$ to be small hence $\|C f\|_{w 2}^{2}$ is disabled.

We can choose W1 to perform the convolution at the edges and not at flat regions since blur is uniform at the flat regions and is given by

$$
W_{1}=1-W_{2}
$$

Here choosing W1 and W2 is not to perform smoothing at the edges, therefore sharp edges will be restored and it will be noisy but not visible, while smoothness will perform at flat regions.

The value of $\mathrm{V}$ can be calculated by working with available noisy image y by removing noise and then finding iteratively i.e. as a function of $\mathrm{K}$ and is given by

$$
\begin{aligned}
& F_{k+1}(u, v)=\beta H^{T} W_{1, k}^{T} W_{1, k} y+ \\
& \left(I-\beta\left(H^{T} W_{1, k}^{T} W H+\alpha C^{T} W_{2, k}^{T} W_{2, k} C\right)\right) f_{k}
\end{aligned}
$$

The performance of spatially adaptive CLS restoration is given in Fig. 11.

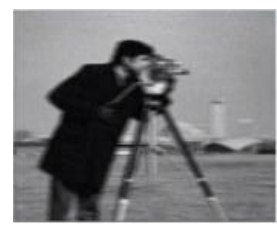

(a)

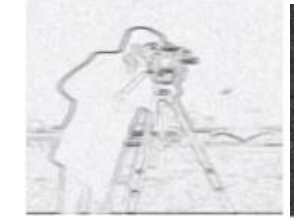

(d)

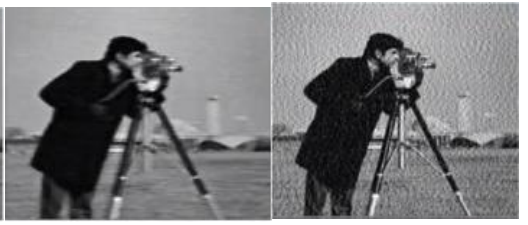

(b)

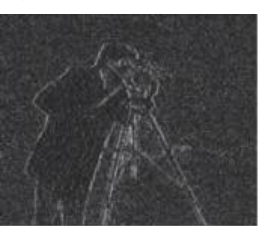

(e)
Fig.11. (a) Motion blurred image with 8pixel and BSNR= 20dB. (b) Iterative Spatially adaptive CLS restored image (c) Iterative CLS restored Image. (d) Visibility Function (e) magnitude error difference of (b) and (c). local activity or alternatively the matrix $\mathrm{V}$ can be updated

By comparing fig.11. (b) and (c), it is observed that there is a good control between noise amplification and sharpness in the adaptively restored image. The edges are considerably well restored while the noise has been supressed. In the iterative CLS filtered image, the edges are sharp but there is a lot of noise amplification. Fig.11. (d) shows the visibility function computed by finding the local variance in the image and smoothness at the edges are disabled so that the grey values of the pixels at the edges are low and at the flat regions the smoothness is enabled and the grey value of the pixels are high. Fig.6. (e) shows the absolute difference between the iterative spatially adaptive CLS filter and iterative non spatially adaptive CLS filters and it is clearly visible that they differ at edges and lot of noise at the flat regions due to the amplification of noise by Iterative CLS filter. Hence it is concluded that spatially adaptive filter is another powerful frame work and its success is depending on appropriate choice of $\mathrm{W} 1$ and $\mathrm{W} 2$.

\section{RESULTS AND COMPARISON}

Frequencies destroyed by the imaging process will actually be infinitely amplified henceforth small amount of noise saturates the inverse filter is shown in Fig.1.and concluding that this filter is more sensitive to noise. Wiener filter is possible to get the satisfactory results but finding powers spectral ratio is not a feasible solution. The excessive noise amplification posed in inverse filtering is existing no longer because of the masking of the spectral zeros, but the image is still somewhat blurred shown in Fig.2. resulting restored image with improvement in the SNR $5.9 \mathrm{~dB}$. This method is nothing but impose a constraint $(\alpha)$ on the solution. In CLS Filters the choice of $\alpha$ is very important, since it gives the trade-off between the noise amplification and smoothness of the filter in Fig.3. it shows that, smaller $\alpha$ results in a noisier but sharper image, while larger $\alpha$ results in smoother but blurrier image. Resulting restored image with improvement in SNR is $6.2 \mathrm{~dB}$. In iterative approaches the restoration process is monitored as it progress, therefore it is possible to terminate the process at any point where the results are satisfactory. In Iterative inverse filter approach For $\mathrm{k} \rightarrow \infty$ or those frequencies $\mathrm{H}(\mathrm{u}, \mathrm{v}) \neq 0$ and the filter acts as inverse filter and for the frequencies $\mathrm{H}(\mathrm{u}, \mathrm{v})=0$, the filter acts as $\mathrm{k} \beta$ and it is concluded that the lower frequencies could be restored faster than the high frequencies. In Iterative LS method It is observation form Fig.6. the general statement by ISNR values from the visual quality, as more the iteration, sharper the restored image. Comparing the two images in Fig.7. restored from the iterative LS filter at $\mathrm{K}=465$ with ISNR $=11.58 \mathrm{~dB}$ and with direct inverse filter, ISNR $=15.50 \mathrm{~dB}$. Restored image by direct inverse filter shows are more pronounced ringing artifacts than the image restored from iterative LS Filter. From Fig.8. It is observed that, noise amplification can be controlled by choosing any intermediate iteration results between $\mathrm{K}=20$ and 50 before the convergence. In Fig.9. It is observed that in Iterative CLS filter ISNR is decreased with the number of iterations 
increases, the edges become sharper and noise multiplication takes place. But still nois amplification is acceptable, compared to the latter approaches. It is showed in Fig.10. Iterative CLS filter is having better ISNR compared to direct CLS filters. Finally Spatially Adaptive restoration results in Fig.11. it is observed that there is a good control between noise amplification and sharpness in the adaptively restored image.

\section{CONCLUTION}

This technical paper discussed several progresses in the area of iterative image deblurring methods. It is presented that iterative approaches are well suitable to image restoration difficulties with linear, spatially invariant distortions (Non Blind Deconvolution) problems. This paper discussed the conventional approaches such as inverse filtering, wiener filtering, connection between the simple iterative algorithms and its extensions to adaptive regularised iterative algorithms and iterative algorithms with higher convergence rates and performance analysis, limitations and Comparison with the experimental results are presented. Since iterative restoration methods have received maturity, the blur identification is open for future work. In practical scenarios, the image subject to real blurs which is nonlinear in nature and restoration of such images required to find unknown blurs from the degraded image itself so-called as Blind Deconvolution Algorithms. Working on Blind Deconvolution problem could be the challenging task for future.

\section{REFERENCES}

1. Andrews, H.C. and Hunt, B.R., Digital Image Restoration, Prentice-Hall, Englewood Cliffs, NJ, 1977.

2. Angel, E.S. and Jain, A.K., Restoration of images degraded by spatially varying point spread functions by a conjugate gradient method, Appl. Opt., 17: 2186-2190, July, 1978.

3. Banham, M. and Katsaggelos, A.K., Digital image restoration, Signal Processing Mag., 14(2), 24-41, Mar., 1997.

4. Mammone, R.J., Computational Methods of Signal Recovery and Recognition, Wiley, 1992.

5. Stark, H., Image Recovery: Theory and Applications, Academic Press, New York, 1987.

6. R. W. Schafer, R. M. Mersereau, and M. A. Richards, "Constrained iterative signal restoration algorithms," Proc. IEEE, vol. 69, pp. 432-450, Apr. 1981.

7. J. L. C. Sanzand T. S. Huang,"Unified Hilbert space approach to iterative least-squares linear signal restoration," I. Opt. Soc. Amer., vol. 73, pp. 1455-1465, Nov. 1983.

8. Y. lchioka and N. Nakajima, "Iterative image restoration considering visibility," J. Opt. Soc. Amer., vol. 71, pp. $983-$ 988, Aug. 1981.

9. H. J. Trussell, "Convergence criteria for iterative restoration methods," IEEE Trans. Acoust., Speech, Signal Process., vol. ASSP-31, pp. 129-136, Feb. 1983.

10. D. C. Youla and H. Webb, "Image restoration by the method of convex projections: Part I-Theory," IEEE Trans. Med. Imaging, vol. MI-1, pp. 81-94, Oct. 1982.

11. M. I. Sezan and H. Stark, "Image restoration by the method of convex projections: Part 2-Applications and numerical results," IEEE Trans. Med. Imaging, vol. MI-I, pp. 95-101, Oct. 1982.
12. A. K. Katsaggelos, J. Biemond, R. M. Mersereau, and R. W. Schafer, "An iterative method for restoring noisy blurred images," Circ. Syst. Signal Proc., vol. 3, pp. 139-160, 1984.

13. A. K. Katsaggelos, Constrained Iterative Restoration Algorithms, Ph.D. Thesis, Georgia Institute of Technology, 1985.

14. A. Levi and H. Stark, "Image restoration by the method of generalized projections with applications to restoration from magnitude,"I. Opt. Soc. Am., vol. 74, pp. 932-943, Sept 1984.

15. R. L. Lagendijk, J. Biemond, and D. E. Boekee, "Regularized iterative image restoration with ringing reduction," IEEE Trans. Acoustics, Speech, Signal Processing, vol. ASSP-36, pp. 1874-1888, Dec. 1988.

16. A. K. Katsaggelos, J. Biemond, R. M. Mersereau, and R. W.Schafer, "Nonstationary iterative image restoration," Proc. IEEE Int. Conf. Acoustics, Speech, Signal Processing, 1985 , pp. 696-699.

17. G. Ross, "Iterative methods in information processing for object restoration," OpticaActa,vol. 29, no. 11, pp. 1523-1542, 1982.

18. A. K. Katsaggelos and R. W. Schafer, "Iterative deconvolution using different distorted versions of an unknown signal," Proc. IEEE lnt. Conf. Acoustics, Speech, Signal Processing.

19. A. K. Katsaggelos, "A general formulation of adaptive iterative image restoration algorithms," Proc. Princeton Conf. on Info. Sciences and Systems, 1986, pp. 42-46.

20. K. Miller, "Least squares methodsfor ill-posed problems with a prescribed bound," SIAM J. Math. Anal., vol. 1, pp. 52-74, Feb. 1970

21. B. R. Hunt, "The application of constrained least-squares estimation to image restoration by digital computer," IEEE Trans. Computers, vol. C-22, pp. 805-812, Sept. 1973.

22. K. Miller, "Least squares methods for ill-posed problems with a prescribed bound," SIAM J. Math. Anal., vol. 1, pp. 52-74, Feb. 1970.

23. Wiener, N. (1949). Extrapolation, interpolation, and smoothing of stationary time series with engineering applications. Journal of the American Statistical Association, 47(258).

24. K. Katsaggelos, J. Biemond, R. M. Mersereau, and R. W. Schafer, "A general formulation of constrained iterative restoration algorithms," Proc. IEEE lnt. Conf. Acoustics, Speech, Signal Processing, 1985, pp. 700-703.

25. R. Marucci, R. M. Mersereau, and R. W. Schafer, "Constrained iterative deconvolution using a conjugate gradient algorithm," Proc. IEEE lnt. Conf. Acoustics, Speech, Signal Processing, 1982, pp. 1845-1848.

26. S. Singh, S. N. Tandon, and H. M. Gupta, "An iterative restoration technique," Signal Processing, vol. 11, pp. 1-11, July 1986.

27. Katsaggelos, A.K., Iterative image restoration algorithm, Opt. Eng., 28(7): 735-748, July, 1989.

28. Efstratiadis, S.N. and Katsaggelos, A.K., Adaptive iterative image restoration with reduced computational load, Opt. Eng., 29: 1458-1468, Dec., 1990.

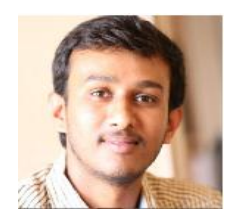

Mahendra B M completed B.E (ECE), M.Tech (Signal Processing) and Pursuing Ph.D from VTU, Karnataka. He has 8 years of teaching experience. Member for professional IEEE. His research interest includes Image restoration and Face

Recognition techniques. 
Dr. Savita Sonoli completed B. E (E\&amp;CE), from BVBCET, Hubli, M.Tech (IE) from NITK, Surathkal, and Ph.D (Embedded Systems) from SK University, Ananthpur. Member for professional bodies, IEEE, ISTE, IEI, ISOI, IAENG, ISCA, ISRASE \&amp; IEAE. 